\title{
Scanning Tunneling Spectroscopy and Luminescent Properties of ZnS:Mn, Cu,Cl Thin Films
}

\author{
G. Neunert, M. Kamiński, B. Susea, T. Dunaj, \\ E. ChIMCZAK* AND M. BERTRANDT-ŻYTKOWIAK \\ Institute of Physics, Poznań University of Technology \\ Nieszawska 13A, 61-021 Poznań, Poland
}

\begin{abstract}
Electrical and luminescent properties of $\mathrm{ZnS}: \mathrm{Mn}, \mathrm{Cu}, \mathrm{Cl}$ thin films were investigated. Combined both studies: scanning tunneling microscopy and scanning tunneling spectroscopy were made. The current and differential conductance versus applied voltage were measured for the $\mathrm{ZnS}: \mathrm{Mn}, \mathrm{Cu}, \mathrm{Cl}$ thin films. Additionally, the spectral and kinetic properties of the electroluminescent cells based on the $\mathrm{ZnS}: \mathrm{Mn}, \mathrm{Cu}, \mathrm{Cl}$ thin films were measured. The maximum of the electroluminescence lies at $586 \mathrm{~nm}$. The electroluminescence was excited by rectangular wave voltage pulses with pulse length from $1 \mu \mathrm{s}$ to $1 \mathrm{~ms}$. It was shown that time dependence of the electroluminescence is well explained assuming energy transfer between monomolecular centres.
\end{abstract}

PACS numbers: 78.60.Fi, 78.66.Hf, 68.37.Ef

\section{Introduction}

During the last decades zinc sulphide doped with manganese luminophors has been widely investigated by many researchers. Destriau [1] in 1936 discovered that a ZnS:Mn powder held in a binder between parallel conducting plates emitted light when ac voltage was applied. Successors of the Destriau devices were dc powered devices [2-4]. They used $\mathrm{Cu}$ doping leads to one of the numerous copper sulphide phases which are conducting or semiconducting. At the end of fifties work on the electroluminescent devices with vacuum deposited semiconductor layer was started by Thornton [5]. The ac thin film devices were pioneered by Inoguchi et al. [6]. In last years numerous papers have been devoted to $\mathrm{ZnS}: \mathrm{Mn}$ nanocrystals [7]. In

*corresponding author; e-mail: chimczak@phys.put.poznan.pl 
the field of luminescence investigations, kinetic measurements are very important since they provide much information concerning the luminescence centres and the mechanism of luminescence in general [8-10]. One of the aims of the papers is description of the electroluminescence of the $\mathrm{ZnS}: \mathrm{Mn}, \mathrm{Cu}, \mathrm{Cl}$ thin films.

The understanding of the nature of electronic properties and atomic structure of materials at the nanometer scale have been the goals of fundamental and applied research.

Due to its extremely high spatial resolution, scanning tunneling microscope (STM) has been widely used to investigation the molecular bonding, nucleation and growth processes and semiconductor morphologies [11, 12].

Since STM images a convolution of the surface electronic and geometric structure, it is often controversial as to whether the contrast is related simply to a difference in geometrical height or electronic effects.

Here we report combined experimental results of the scanning tunneling microscopy and spectroscopy (STM/STS) on ZnS:Mn, Cu,Cl films at room temperature. Tunneling experiment provide complimentary information on the electronic structure because discrete occupied and unoccupied energy levels are probed separately.

\section{Experimental}

The $\mathrm{ZnS}: \mathrm{Mn}, \mathrm{Cu}, \mathrm{Cl}$ thin films were produced onto a glass substrate by thermal evaporation from quartz crucibles which contained the mixture of powder $\mathrm{ZnS}: \mathrm{Mn}(70 \%)$ and powder $\mathrm{CuCl}(30 \%)$. The powdered zinc sulphide has been activated with manganese concentration of $3 \mathrm{wt} \%$. In order to obtain electroluminescent cells the outer aluminum electrodes were evaporated. The electroluminescence was excited by rectangular voltage pulses with pulse length from $1 \mu \mathrm{s}$ to $1 \mathrm{~ms}$. Light emission is observed from the surface reproducing exactly the aluminum electrode.

The Cryo SXM (OMICRON) scanning tunneling microscope was used to study the topography and spectroscopic properties of the $\mathrm{ZnS}: \mathrm{Mn}, \mathrm{Cu}, \mathrm{Cl}$ thin films. Imaging was performed in constant current mode $(1.0 \mathrm{nA})$ at sample bias $+1.0 \mathrm{~V}$, using a $W$ tip. The scaling of the microscope was calibrated on a highly oriented pyrolytic graphite surface.

\section{Results and discussion}

A semiconductor with $n$-type dopands has donor levels and the thermal excitation of electrons from donor levels populates the bottom of the conduction band.

For a semiconductor-insulator-metal (SIM) junction with $n$-type semiconductor, the Fermi level is close to the donor level $E_{\mathrm{D}}$ of the semiconductor when 
$U_{\text {bias }}=0$. Depending upon the sign and magnitude of the bias voltage, the STM current of doped semiconductors may involve not only the bottom of the conduction band but also the donor levels lying in the band gap. In addition, the wave functions of the donor levels are spatially localized in the regions around the donor, unlike those of the valence and conduction band levels which are delocalized. Therefore STM images of doped semiconductor can exhibit local imperfections.

Figure 1 shows a typical STM image of ZnS:Mn,Cu,Cl thin films measured at room temperature.

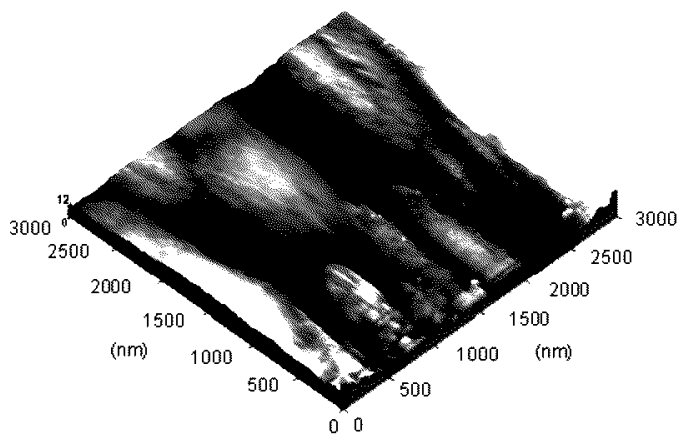

Fig. 1. STM image of $\mathrm{ZnS}: \mathrm{Mn}, \mathrm{Cu}, \mathrm{Cl}$ thin films measured at room temperature. The image size is $3 \times 3 \mu \mathrm{m}$ sample bias voltage and tunneling current are $\pm 0.5 \mathrm{~V}$ and $0.4 \mathrm{nA}$, respectively.

When a bias voltage is applied through the SIM junction the energy levels of the semiconductor bands bend depending on the bias polarity. At a large negative bias, the band bending of an $n$-type semiconductor may populate the bottom position of the conduction band at the surface, so that electrons may also flow from the bottom of the conduction band to the tip. When the magnitude of the negative bias is greater than $E_{\mathrm{g}} / 2$ so that $E_{V}+e\left|U_{\text {bias }}\right|>E_{\mathrm{F}}$, the electrons in the valence band of the sample tunnel into the tip and we can observe large current for strongly negative bias.

The results of STS measurements are presented by plotting the current voltage characteristic, or more commonly tunneling conductance vs. bias voltage for $\mathrm{ZnS}: \mathrm{Mn}, \mathrm{Cu}, \mathrm{Cl}$ thin film. The plot of the difference conductance is proportional to electronic density of states $n(E)$. Figure 2 shows the function $\mathrm{d} I / \mathrm{d} U$ versus $U$ characteristics for $\mathrm{ZnS}: \mathrm{Mn}, \mathrm{Cu}, \mathrm{Cl}$ thin film surface.

Tunneling conductance vs. bias voltage has been suggested as a qualitative measurement of the local electronic density of states. Positive values of the bias voltage represent tunneling from the tip into both the donor level and the unoccupied sample states. While negative values correspond to tunneling from occupied donor level for small bias and the valence band top for large bias into unoccupied tip states. For the $\mathrm{ZnS}: \mathrm{Mn}, \mathrm{Cu}, \mathrm{Cl}$ surface two occupied states at $-0.3 \mathrm{~V}$ and $-0.4 \mathrm{~V}$ 


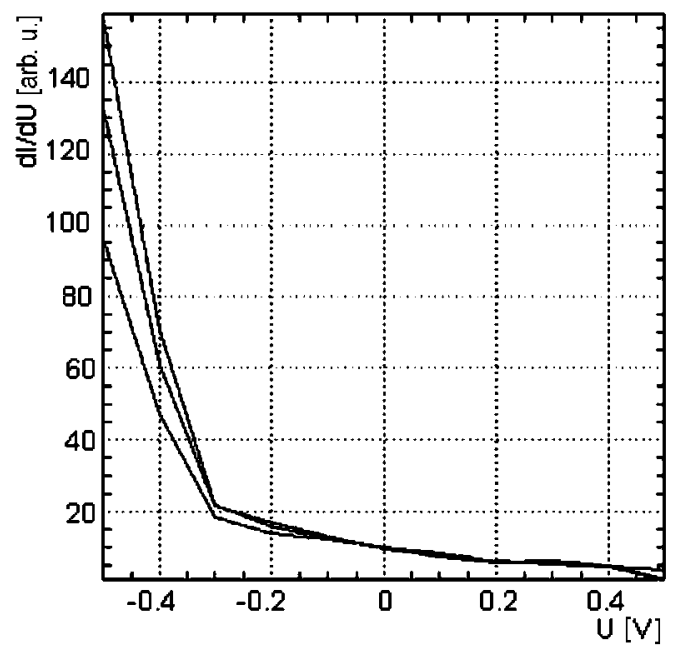

Fig. 2. Tunneling conductance vs. bias voltage for $\mathrm{ZnS}: \mathrm{Mn}, \mathrm{Cu}, \mathrm{Cl}$ thin film.

are seen, but unoccupied sample states are not detected for this voltage regime. Rather, not a clear gap is seen, suggesting that $\mathrm{ZnS}: \mathrm{Mn}, \mathrm{Cu}, \mathrm{Cl}$ local area are not semiconducting but rather metallic for small bias voltage $\left(<E_{\mathrm{g}} / 2\right)$.

The $\mathrm{ZnS}: \mathrm{Mn}, \mathrm{Cu}, \mathrm{Cl}-\mathrm{Al}$ thin film cells exhibit electroluminescence for both $\mathrm{dc}$ and ac supply voltages. The cells emit characteristic of manganese activator yellow-orange electroluminescence (EL) with maximum at $586 \mathrm{~nm}$ (Fig. 3). The electroluminescence spectrum is almost restricted to the range of spectral sensitivity of the human eye.

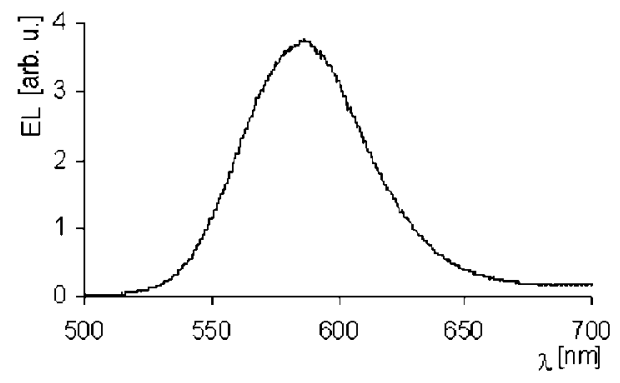

Fig. 3. Electroluminescence spectrum of the cell investigated.

When a long-time rectangular voltage pulses are supplied to the investigated cells, the electroluminescence rises during the excitation pulse and decays immediately after the end of the pulse (Fig. 4).

Very interesting phenomenon is observed for the short pulse excitation. In this case the maximum of the electroluminescence appears at the time $t_{\mathrm{m}}$ after the end of the exciting pulse (Fig. 5). 


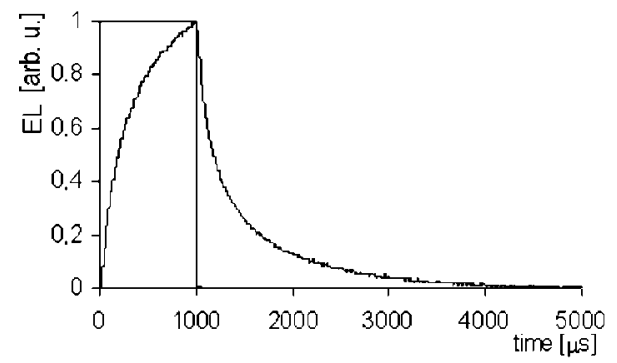

Fig. 4. Time dependence of the electroluminescence excited by a rectangular pulse of $1 \mathrm{~ms}$.

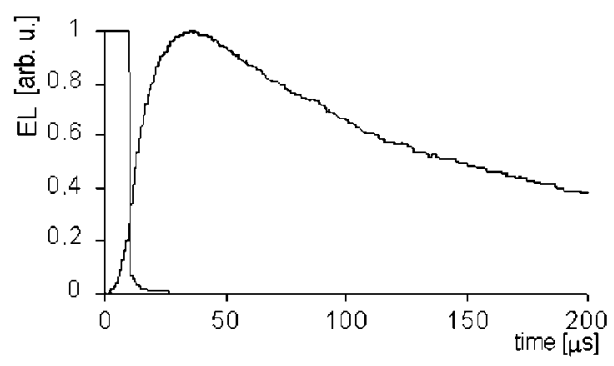

Fig. 5. Time dependence of the electroluminescence excited by a rectangular pulse of $10 \mu \mathrm{s}$.

Another interesting phenomenon is the non-typical behavior of the electroluminescence observed during the exciting pulse. As is seen (Fig. 6) the time dependences are considerably different from that for direct excited luminescence described by equation

$$
I=G\left[1-\exp \left(-t / \tau_{e}\right)\right]
$$

during the exciting pulse and

$$
I=I_{0} \exp \left(-t / \tau_{\mathrm{e}}\right)
$$

after the end of the exciting pulse. In the equations $G$ is generation rate and $\tau_{\mathrm{e}}$ is lifetime of emitting centres. During the first microseconds the electroluminescence is practically not seen. In order to explain these phenomena we assumed energy transfer between two monomolecular centres [10, 13, 14].

If we assume that each part $\mathrm{d} t$ of the exciting pulse $T$ produces the elemental curve

$$
\mathrm{d} I=\frac{G \mathrm{~d} t}{\tau_{\mathrm{e}}-\tau_{\mathrm{t}}}\left[\exp \left(-t / \tau_{\mathrm{e}}\right)-\exp \left(-t / \tau_{\mathrm{t}}\right)\right]
$$

then we obtain the time dependence during the exciting pulse

$$
I=\frac{G}{\tau_{\mathrm{e}}-\tau_{\mathrm{t}}}\left\{\tau_{\mathrm{e}}\left[1-\exp \left(-t / \tau_{\mathrm{e}}\right)\right]-\tau_{\mathrm{t}}\left[1-\exp \left(-t / \tau_{\mathrm{t}}\right)\right]\right\}
$$




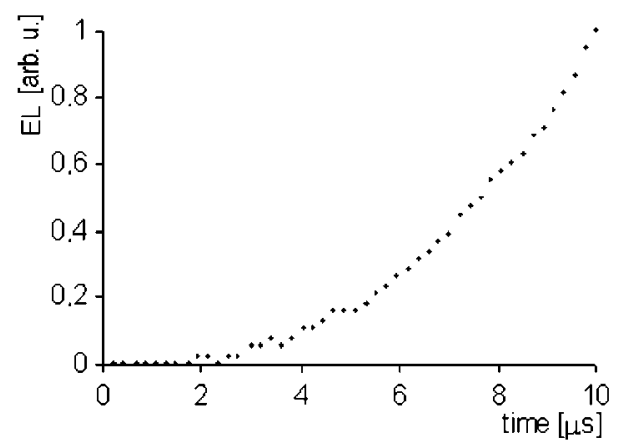

Fig. 6. Time dependence of the electroluminescence during the exciting rectangular pulse of $10 \mu \mathrm{s}$.
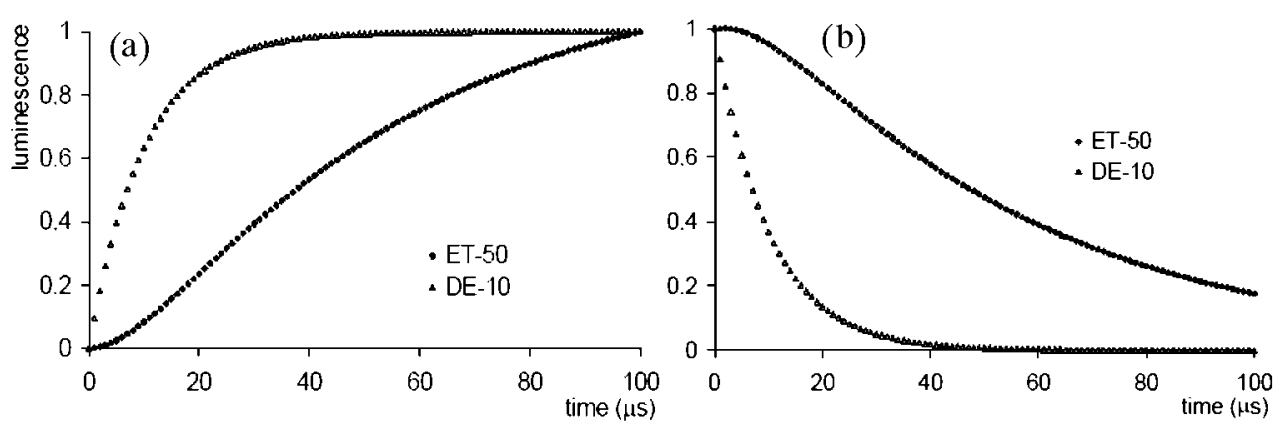

Fig. 7. Theoretical time dependence of luminescence during (a) and after the end (b) of the exciting rectangular pulse $T$ plotted for energy transfer (ET) and direct excitation (DE) for $\tau_{\mathrm{e}}=10 \mu \mathrm{s}$ and $\tau_{\mathrm{t}}=50 \mu \mathrm{s}$.

and

$$
\begin{aligned}
I= & \frac{G}{\tau_{\mathrm{e}}-\tau_{\mathrm{t}}}\left\{\tau_{\mathrm{e}}\left[1-\exp \left(-T / \tau_{\mathrm{e}}\right)\right] \exp \left(-t / \tau_{\mathrm{e}}\right)\right. \\
& \left.-\tau_{\mathrm{t}}\left[1-\exp \left(-T / \tau_{\mathrm{t}}\right)\right] \exp \left(-t / \tau_{\mathrm{t}}\right)\right\}
\end{aligned}
$$

after the end of the exciting pulse, where $\tau_{\mathrm{t}}$ is lifetime of transferring centres. Figure 7 a shows the theoretical time dependences of luminescence during the exciting pulse described by Eq. (4) (ET-50) and Eq. (1) (DE-10). The dependences after the end of the exciting rectangular pulse are shown in Fig. 7b. In the both cases the direct excitation mechanism (DE) cannot be used to describe the experimental data. As is seen, energy transfer mechanism (ET) very well explains the behavior of the manganese electroluminescence during (Fig. 7a) as well as after (Fig. 7b) the exciting rectangular pulse. 


\section{Summary}

Using scanning tunneling spectroscopy we detected the localized state near the Fermi level on the $\mathrm{ZnS}: \mathrm{Mn}, \mathrm{Cu}, \mathrm{Cl}$ surface. These data reveal two occupied states at $-0.3 \mathrm{~V}$ and $-0.4 \mathrm{~V}$ but unoccupied sample states are not detected for this voltage regime.

The $\mathrm{ZnS}: \mathrm{Mn}, \mathrm{Cu}, \mathrm{Cl}-\mathrm{Al}$ thin film cells emit characteristic of manganese activator yellow-orange light. For a long-time excitation the electroluminescence rises during the exciting pulse and decays immediately after the end of the pulse. For the short pulse excitation the maximum of the electroluminescence appears after the end of the exciting pulse. Energy transfer mechanism very well explains the behavior of the time dependence of the electroluminescence.

This work was supported by Poznań University of Technology Research Program PB 62-198/03-BW.

\section{References}

[1] G. Destriau, J. Chim. Phys. 33, 587 (1936).

[2] A.G. Fischer, J. Electrochem. Soc. 109, 1043 (1962).

[3] A. Vecht, J. Lumin. 7, 213 (1973).

[4] N.A. Vlasenko, J.A. Popkov, Opt. Spektrosk. 8, 39 (1960).

[5] W.A. Thornton, J. Appl. Phys. 33, 3045 (1962).

[6] T. Inoguchi, M. Takeda, Y. Kakihara, Y. Nakata, M. Yoshida, Sid Intern. Symp. Dig. 86, (1974).

[7] T. Kubo, T. Isobe, M. Senna, J. Lumin. 99, 39 (2002).

[8] S. Tanaka, H. Kobayashi, H. Sasakura, Y. Hamakawa, J. Appl. Phys. 47, 5391 (1976).

[9] E. Chimczak, J.W. Allen, J. Phys. D 18, 951 (1985).

[10] M. Bertrandt-Żytkowiak, E. Chimczak, Thin Solid Films 256, 136 (1995).

[11] H. Wang, C. Zeng, Q. Li, B. Wang, J. Yang, J.G. Hou, Q. Zhu, Surf. Sci. 442, LI024 (1999).

[12] A.W. Dunn, E.D. Svensson, C. Dekker, Surf. Sci. 498, 237 (2001).

[13] E. Chimczak, J. Lumin. 39, 247 (1988).

[14] E. Chimczak, Z. Phys. B 72, 211 (1988). 